\title{
STATISTICS AND CHARACTERIZATION OF MATRICES BY DETERMINANT AND TRACE
}

\author{
EMRE ALKAN AND EKIN SILA YÖRÜK
}

\begin{abstract}
Answering a question of Erdös, Komlós proved in 1968 that almost all $n \times n$ Bernoulli matrices are nonsingular as $n \rightarrow \infty$. In this paper, we offer a new perspective on the question of Erdös by studying $n \times n$ matrices with prime number entries in an almost all sense. Precisely, it is shown that, as $x \rightarrow \infty$, the probability of randomly choosing a nonsingular $n \times n$ matrix among all $n \times n$ matrices with prime number entries that are $\leq x$ is 1 . If $A$ is a unitary matrix, then it is well-known that $|\operatorname{det} A|=1$. However, the converse is far from being true. As a remedy of this defect, we search for necessary and sufficient conditions for being a unitary matrix by teaming up determinant with trace. In this way, we are led to simple characterizations of unitary matrices in the set of normal matrices. The question of which nonsingular commuting complex matrices with real eigenvalues have the same characteristic polynomial is formulated via determinant and trace conditions. Finally, through a study of eigenvectors, we obtain new characterizations of hermitian and normal matrices. Our approach to proving these results benefits from a modular interpretation of nonsingularity and the spectral theorem for normal operators together with equality cases of classical inequalities such as the arithmetic-geometric mean inequality and the CauchySchwarz inequality.
\end{abstract}

\section{INTRODUCTION}

Let $A$ be a square matrix of size $n$ with complex entries. Two important quantities that are associated to $A$ are the determinant and trace, denoted as $\operatorname{det} A$ and $\operatorname{tr}(A)$, respectively. If $\lambda_{1}, \ldots, \lambda_{n}$ are the eigenvalues of $A$, then

$$
\operatorname{det} A=\prod_{i} \lambda_{i} \text { and } \operatorname{tr}(A)=\sum_{i} \lambda_{i}
$$

where both of them are symmetric functions of the $\lambda_{i}$. Despite the well-known fact that determinant and trace are both invariants under similarity transformations, the converse almost never holds. One of the motivations of this paper is to repair this defect for specific classes of matrices and further obtain characterizations of them in terms of statements involving only determinant and trace. To quote another well-known fact, note that the vanishing of determinant characterizes the singularity of a matrix. In this regard, singular matrices are expected to be statistically rare among all matrices. For this expectation to be meaningful, we need to put constraints on the entries of the matrices under consideration. A natural and simple setting would be to focus on $n \times n$ matrices with nonnegative integer entries that are $\leq x$ (here we are assuming that $x \geq 1$ ). Answering a question of Erdös, Komlós [11], [12] proved in this setting that the probability of randomly choosing a singular matrix tends to 0 as $n \rightarrow \infty$. In particular, for the first interesting case when $x=1$, matrices with 0,1 entries are

2010 Mathematics subject classification. 15B36, 15A57, 15A42, $15 \mathrm{~A} 18$.

Key words and phrases: Matrices with prime number entries, almost all, determinant, trace, unitary matrix, hermitian matrix, normal matrix. 
called Bernoulli matrices. An arithmetic question on unimodular matrices was nicely treated by Dănescu, Vâjâitu and Zaharescu [4]. There are alternative ways to quantify the rareness of singular matrices. For fixed $n$, one could as well look at matrices whose entries are $\leq x$ and belong to a thin subset of positive integers, such as the set of prime numbers, with the hope of showing that the singular ones among them are still rare as $x \rightarrow \infty$. This would then give a new direction on the question of Erdös. Our main result below confirms the desired statistical expectation in an almost all sense among matrices all of whose entries belong to the set of prime numbers. We should mention that almost all type results are common in number theory. Recall that a property $\mathcal{P}$ holds for almost all positive integers, if

$$
\lim _{x \rightarrow \infty} \frac{\mathcal{P}(x)}{x}=1,
$$

where $\mathcal{P}(x)$ is the number of integers that are $\leq x$ and have the property $\mathcal{P}$. A classical result of Hardy and Ramanujan [9] states that almost all positive integers $n$ have about $\log \log n$ prime factors. A celebrated theorem of Erdös and Kac [7] extends this phenomenon to the values of a wide class of arithmetic functions (for a characterization of additive arithmetic functions with continuous limiting distributions, see [6]). A special case of their striking discovery shows that the number of prime factors of a positive integer $n$ behaves like a Gaussian normal distribution with mean and variance both equal to $\log \log n$. We can now state the main result.

Theorem 1. Asymptotically, almost all $n \times n$ matrices with prime number entries are nonsingular. Precisely, if $M_{n}(x)$ is the number of nonsingular $n \times n$ matrices with prime number entries that are $\leq x$ and $\pi(x)$ is the number of prime numbers that are $\leq x$, then

$$
\lim _{x \rightarrow \infty} \frac{M_{n}(x)}{\pi(x)^{n^{2}}}=1
$$

Consequently, as $x \rightarrow \infty$, the probability of randomly choosing a nonsingular $n \times n$ matrix among all $n \times n$ matrices with prime number entries that are $\leq x$ is 1 .

Some remarks on Theorem 1 are now in order. First by the prime number theorem (see Chap. 18 of [5])

$$
\pi(x) \sim \frac{x}{\log x} \sim \operatorname{li}(x)=\int_{2}^{x} \frac{1}{\log t} d t
$$

as $x \rightarrow \infty$. Thus Theorem 1 can be rephrased as

$$
M_{n}(x) \sim\left(\frac{x}{\log x}\right)^{n^{2}}
$$

as $x \rightarrow \infty$. Recall that a set of positive integers $\mathcal{A}$ has asymptotic density 0 if

$$
\lim _{x \rightarrow \infty} \frac{\mathcal{A}(x)}{x}=0,
$$

where $\mathcal{A}(x)$ is the number of integers in $\mathcal{A}$ that are $\leq x$. Note that the set of prime numbers has asymptotic density 0 and more generally, a set with asymptotic density 0 can be viewed as a thin subset of positive integers. It would be an interesting task to obtain analogs of Theorem 1 for matrices with entries coming from other thin subsets of integers. It turns out that, by adapting our method of proof of Theorem 1, such a task can be undertaken if the set of integers, where the entries of the matrices under consideration belong to, is sufficiently well distributed over arithmetic progressions. In particular, the prime number theorem for arithmetic progressions (see Chap. 20 of [5]) is needed in the proof of Theorem 1, 
thereby exploiting the fact that prime numbers are asymptotically equally distributed among arithmetic progressions which admit infinitely many of them.

Matrices possessing more structure such as symmetry are ubiquitous throughout linear algebra (and most of the rest of mathematics as well). These include representations arising from unitary, hermitian, skew-hermitian and normal operators which indeed constitute a foundation for the mathematical formulation of quantum mechanics (see Chap. 3 of [8]). As a result of the impact of such operators on applied problems, alternative characterizations of them were obtained and used (see Chap. 7 of [13]). Recall that $A$ is real unitary precisely when

$$
A A^{t}=I
$$

where $A^{t}$ is the transpose of $A$. An obvious necessary condition for being real unitary is then

$$
|\operatorname{det} A|=1 \text {. }
$$

However, this condition is far from being a sufficient condition. An elegant necessary and sufficient condition can be given by teaming determinant with trace. Although it may still not be clear which matrix should be associated with the trace, one is able to infer a slightly stronger statement. For the notation, we let $\langle.,$.$\rangle be a positive definite bilinear form (or$ scalar product) on $\mathbb{R}^{n}$.

Theorem 2. Let $A$ be a matrix of size $n$ with real entries. Then $A$ is similar (with respect to an orthonormal basis arising from $\langle.,$.$\rangle ) to a block matrix B$ consisting of only $2 \times 2$ and $1 \times 1$ blocks of the forms

$$
\left[\begin{array}{cc}
\cos \theta & -\sin \theta \\
\sin \theta & \cos \theta
\end{array}\right], \quad[1], \quad[-1]
$$

on the main diagonal (so that other entries of $B$ are 0 ) if and only if

$$
|\operatorname{det} A|=1 \text { and } \operatorname{tr}\left(A A^{t}\right)=n \text {. }
$$

Our proof of Theorem 2 rests on the relation between determinant and trace via the arithmeticgeometric mean inequality,

$$
\frac{1}{n} \sum_{i=1}^{n} x_{i} \geq\left(\prod_{i=1}^{n} x_{i}\right)^{\frac{1}{n}}
$$

for nonnegative real numbers $x_{1}, \ldots, x_{n}$, equality being possible only when all $x_{i}$ are the same. For a complex unitary matrix $A$ satisfying

$$
A A^{*}=I,
$$

where $A^{*}$ is the conjugate transpose of $A$, a similar characterization can be given as a bonus by making the necessary modifications in Theorem 2 . This time we let $\langle.,$.$\rangle be a positive$ definite hermitian form on $\mathbb{C}^{n}$.

Theorem 3. Let $A$ be a matrix of size $n$ with complex entries. Then $A$ is similar (with respect to an orthonormal basis arising from $\langle.,$.$\rangle ) to a diagonal matrix B$ consisting of entries of the form $e^{i \theta}$ on the main diagonal if and only if

$$
|\operatorname{det} A|=1 \text { and } \operatorname{tr}\left(A A^{*}\right)=n \text {. }
$$


The conditions of being hermitian and normal for a matrix $A$ are given by

$$
A=A^{*} \text { and } A A^{*}=A^{*} A,
$$

respectively. Consequently, unitary and hermitian matrices are normal, though hermitian and normal matrices don't possess group structure, a key property shared by unitary matrices. Furthermore, unitary matrices are characterized by preserving unit vectors (see p. 189 of [13]). The following result keeps the essence of Theorem 2 and Theorem 3 by providing a new characterization of complex unitary matrices when they are viewed as members of the collection of normal matrices.

Corollary 1. Assume that $A$ is a normal matrix of size $n$ with complex eigenvalues $\lambda_{1}, \ldots, \lambda_{n}$. Then $A$ is complex unitary if and only if

$$
|\operatorname{det} A|=1 \text { and } \sum_{i}\left|\lambda_{i}\right|=n .
$$

Indeed it is possible to formulate the unitary condition solely as an equation involving determinant and trace.

Theorem 4. Let $N$ be a nonsingular complex matrix of size $n$. Then there exists a positive number $c$ such that $c N$ is unitary if and only if the equality

$$
\frac{\operatorname{tr}\left(N N^{*}\right)}{n}=|\operatorname{det} N|^{\frac{2}{n}}
$$

holds.

Assume for the moment that $A$ and $B$ are similar matrices. Then $B=P^{-1} A P$ holds for some nonsingular matrix $P$. We certainly have then $\operatorname{det} A=\operatorname{det} B, \operatorname{tr}(A)=\operatorname{tr}(B)$ and $A, B$ have the same characteristic polynomial so the same eigenvalues counting multiplicity. Again, in general, converses of these implications are not true. A better measure of similarity might go through the comparison of Jordan normal forms of $A$ and $B$ (see Chap. 11 of [13]). Observe that the computation of determinant and trace uses data only from the main diagonal of the Jordan normal form but not from the actual blocks appearing in the Jordan normal form. Therefore, we can't hope to deduce the similarity of matrices in an obvious way from determinant and trace conditions. Despite of this, it is possible to salvage the conclusion that two matrices have the same eigenvalues counting multiplicity out of a combination of determinant and trace conditions.

Theorem 5. Let $A$ and $B$ be nonsingular commuting complex matrices with real eigenvalues. Then $A$ and $B$ have the same characteristic polynomial if and only if

$$
\operatorname{det} A=\operatorname{det} B \text { and }(\operatorname{tr}(A B))^{2}=\operatorname{tr}\left(A^{2}\right) \operatorname{tr}\left(B^{2}\right) .
$$

It is interesting to note how the trace condition in (1.2) mimics the equality case of the Cauchy-Schwarz inequality. The set of eigenvalues of an operator is called the spectrum of that operator so that in the case of Theorem $5, A$ and $B$ would also have the same spectrum if the hypotheses are satisfied. Let us have a brief digression to generate plenty of examples of nonsingular commuting matrices with real eigenvalues as in Theorem 5 . To this end, first start with two Jordan normal forms, say $J_{1}$ and $J_{2}$, subject to the following conditions. If $J_{1}$ has a block of the form $\lambda I+N$, where $N$ is the nilpotent part consisting of 1 's above the main diagonal, then $J_{2}$ either has a block of the same form, namely has a block of the form $\mu I+N$, or it has a diagonal block of the form $\mu I$ having the same size. Note that $\lambda I+N$ commutes 
with $\mu I+N$ and $\mu I$. Consequently, assuming also that the corresponding blocks of $J_{1}$ and $J_{2}$ appear at the same places on the main diagonal, we see that $J_{1} J_{2}=J_{2} J_{1}$. Next take any nonsingular matrix $P$. Then let $A=P^{-1} J_{1} P$ and $B=P^{-1} J_{2} P$ so that $A$ and $B$ commute as $J_{1}$ and $J_{2}$ commute. Finally, the eigenvalues of $A, B$ are the $\lambda$ 's and $\mu$ 's so we may choose them to be nonzero real numbers. This guarantees that $A$ and $B$ are nonsingular as well. Having generated examples, we may assume that $A, B$ are nonsingular commuting matrices with real eigenvalues satisfying (1.2). Thus Theorem 5 applies and shows that $A$ and $B$ have the same eigenvalues with the same multiplicities. We finish this digression by a discussion of some extra conditions imposed on $A$ and $B$ which would force them to be similar. In addition to the above conditions, assume that the geometric multiplicity of each eigenvalue of $A, B$ are the same. This means that for each common eigenvalue $\lambda$,

$$
\operatorname{dim} \operatorname{Ker}(A-\lambda I)=\operatorname{dim} \operatorname{Ker}(B-\lambda I)
$$

holds, where $\operatorname{dim} \operatorname{Ker}(A-\lambda I)$ and $\operatorname{dim} \operatorname{Ker}(B-\lambda I)$ denote the dimensions of the kernel of the corresponding operators. Moreover, if $(t-\lambda)^{m_{1}}$ and $(t-\lambda)^{m_{2}}$ are the factors, corresponding to $\lambda$, in the minimal polynomials of $A$ and $B$, respectively, then in either of the cases when

$$
\max \left(m_{1}, m_{2}\right) \leq 2
$$

for all $\lambda$ or

$$
m_{1}=m_{2} \geq m-3
$$

for all $\lambda$, where $m$ is the multiplicity of $\lambda$ (so that $(t-\lambda)^{m}$ is the factor corresponding to $\lambda$ in the common characteristic polynomial of $A, B)$, one can infer that $A, B$ are similar. To justify this first assume that $\max \left(m_{1}, m_{2}\right) \leq 2$. Consider the Jordan normal forms of $A$ and $B$. All blocks involving $\lambda$ must have size at most 2 in both of the Jordan normal forms of $A, B$. As the geometric multiplicities are the same, the total number of 1's appearing in all of the blocks involving $\lambda$ must be the same (observe that the total number of 1's is equal to the multiplicity of $\lambda$ minus the geometric multiplicity of $\lambda$ ) in both of the Jordan normal forms of $A, B$. This means that the number of $2 \times 2$ blocks should be the same in both of the Jordan normal forms of $A, B$ for every common eigenvalue $\lambda$. Therefore, the Jordan normal forms of $A, B$ are similar and it follows that $A, B$ are similar. For the other case when $m_{1}=m_{2} \geq m-3$, if there is a block of size $m-3$ corresponding to $\lambda$ in both of the Jordan normal forms of $A, B$, then the remaining part can decompose as a single $3 \times 3$ or one $2 \times 2$ and one $1 \times 1$ or three $1 \times 1$. Again the total number of 1 's must be the same and blocks in the Jordan normal forms of $A, B$ are identical except possibly their places on the main diagonal. Thus $A$ and $B$ are forced to be similar. If there is a block of size $>m-3$ corresponding to $\lambda$ in both of the Jordan normal forms of $A, B$, then it can be shown as above that $A$ and $B$ are again similar.

The spectral theorem holds for normal matrices (see p. 227 of [13]) which makes them amenable to diagonalization with respect to an orthonormal basis consisting of eigenvectors. A characterization of normal operators were obtained by Hoffman and Taussky [10]. In this connection, it is possible to give a simple and elegant treatment of hermitian and normal matrices separately by focusing on their eigenvectors. The case of hermitian matrices is handled in the next theorem.

Theorem 6. Assume that $A$ is a complex matrix with real eigenvalues. Then $A$ is hermitian if and only if $A$ and $A^{*}$ have the same eigenvectors.

For normal matrices there is an analogous criterion in terms of eigenvectors. 
Theorem 7. Let $A$ be a complex matrix. Then $A$ is normal if and only if $A A^{*}$ and $A^{*} A$ have the same eigenvectors corresponding to every common eigenvalue of $A A^{*}$ and $A^{*} A$.

\section{A MODULAR INTERPRETATION OF NONSINGULARITY}

Let $A=\left(a_{i j}\right)$ be a given $n \times n$ matrix with positive integer entries. For any prime number $p$, let

$$
A_{p}=\left(\overline{a_{i j}}\right)
$$

be the $n \times n$ matrix obtained from $A$ by reducing entries of $A$ modulo $p$. Thus $A_{p}$ can be regarded as a matrix over the field

$$
\mathbb{Z}_{p}=\{\overline{0}, \overline{1}, \ldots, \overline{p-1}\}
$$

of congruence classes modulo $p$. The following characterization of the nonsingularity of $A$ will be one of the key ingredients in the proof of Theorem 1.

Theorem 8. (Modular interpretation of nonsingularity) Let $A$ be a matrix of size $n$ with positive integer entries. Then $A$ is nonsingular if and only if $A_{p}$ is nonsingular for some prime number $p$.

To prove Theorem 8 , first let $A=\left(a_{i j}\right)$. Then by the well-known determinant formula,

$$
\operatorname{det} A=\sum_{\sigma \in S_{n}}(-1)^{\epsilon(\sigma)} a_{1, \sigma(1)} \ldots a_{n, \sigma(n)},
$$

where $\sigma$ ranges over all permutations in the symmetric group $S_{n}$ and $\epsilon(\sigma)$ gives the parity of the permutation which is 0 or 1 according to when $\sigma$ is an even or odd permutation, respectively. Now $\operatorname{det} A$ is an integer and if $\overline{\operatorname{det} A} \in \mathbb{Z}_{p}$ denotes the reduction of $\operatorname{det} A$ modulo $p$, then we see from the above formula that

$$
\overline{\operatorname{det} A}=\sum_{\sigma \in S_{n}}(-1)^{\epsilon(\sigma)} \overline{a_{1, \sigma(1)}} \ldots \overline{a_{n, \sigma(n)}}=\operatorname{det} A_{p}
$$

as $A_{p}=\left(\overline{a_{i j}}\right)$. If $A_{p}$ is nonsingular for some prime $p$, then $\operatorname{det} A_{p} \neq \overline{0}$ and this forces $\operatorname{det} A \neq 0$. Therefore, $A$ has to be nonsingular. Conversely, if $A$ is nonsingular, then $\operatorname{det} A$ is a nonzero integer. Let $p$ be a prime number not dividing $\operatorname{det} A$. Then $\overline{\operatorname{det} A} \neq \overline{0}$ in $\mathbb{Z}_{p}$ and this forces $\operatorname{det} A_{p} \neq \overline{0}$. Thus $A_{p}$ is nonsingular for some prime number $p$. This completes the verification of Theorem 8. It is worth remarking that Theorem 8 builds on the simple idea of reading entries modulo a prime number. Reading entries modulo prime numbers is a fruitful idea in other areas of mathematics such as the local to global principles in the theory of Diophantine equations which motivated most of the developments in algebraic number theory and class field theory (see Chaps. 2, 5, 6 of [14] and first part of [16] for the Hasse-Minkowski theorem). This idea also led to the theory of supersingular primes by studying elliptic curves $p$-adically. For results on the Fourier coefficients of cusp forms associated to elliptic curves using the distribution of supersingular primes, see [2] and [3] which form part of the first author's doctoral thesis [1]. Thus, inspired by these applications, there is good motivation to name this section and Theorem 8 as above.

In conclusion, to show that such a matrix $A$ as in Theorem 8 is nonsingular, it is enough to find a prime number $p$ and show that $A_{p}$ is nonsingular over $\mathbb{Z}_{p}$. It makes sense, of course, to search for the smallest prime number $p$ that does the job. Let us therefore call the smallest 
prime number $p$ such that $A_{p}$ is nonsingular, the complexity of $A$. Note that the complexity of the $3 \times 3$ matrix

$$
A=\left[\begin{array}{ccc}
2 & 4 & 6 \\
15 & 3 & 12 \\
25 & 50 & 101
\end{array}\right]
$$

is 5 as $A_{2}$ and $A_{3}$ are singular but $A_{5}$ is not as $\operatorname{det} A_{5}=\overline{1}$ in $\mathbb{Z}_{5}$. It is also clear from the proof of Theorem 8 that if $A$ is nonsingular, then the complexity of $A$ is the smallest prime number not dividing $\operatorname{det} A$. Although our modular interpretation of nonsingularity is theoretically interesting, verifying that a given matrix $A$ is nonsingular with the help of this principle can be arbitrarily difficult. This is due to the fact that the complexity is unbounded. Indeed the unboundedness even holds for matrices having prime number entries.

Theorem 9. Let $N$ be a positive integer and $n \geq 2$. Then there exist infinitely many $n \times n$ matrices with prime number entries whose complexity are all $>N$.

Observe that Theorem 9 is not true when $n=1$ as the complexity of a $1 \times 1$ matrix $[p]$, where $p$ is an odd prime, is always 2. We may now give the proof of Theorem 9 . Let $p_{k+1}$ be the least prime that is $>N$ and put

$$
P=\prod_{i=1}^{k} p_{i}
$$

where $2=p_{1}<3=p_{2}<\cdots<p_{i}<\ldots$ is the sequence of primes in increasing order. Then consider the progression $P m+1$, where $m$ ranges over positive integers. By Dirichlet's theorem, there are infinitely many prime numbers belonging to this progression. We prove by induction over $n \geq 2$ that there exist infinitely many $n \times n$ matrices with prime number entries in the progression $P m+1$ whose complexity are all $>N$. For the base case of the induction, let $A=\left(a_{i j}\right)$ be a $2 \times 2$ matrix with prime number entries in the progression $P m+1$. Then note that

$$
A_{p_{i}}=\left[\begin{array}{ll}
\overline{1} & \overline{1} \\
\overline{1} & \overline{1}
\end{array}\right]
$$

and $A_{p_{i}}$ is singular for all $1 \leq i \leq k$. Moreover, we have $\operatorname{det} A=a_{11} a_{22}-a_{21} a_{12}$ and keeping $a_{12}, a_{21}, a_{22}$ fixed, we may vary $a_{11}$ along the progression $P m+1$ to guarantee that $\operatorname{det} A \neq 0$. Thus for each such choice of $a_{11}$, the corresponding $A$ is nonsingular and its complexity is $\geq p_{k+1}>N$. Clearly, there are infinitely many such $A$ as there are infinitely many choices for $a_{11}$. This settles the base case. For the inductive step, let $A=\left(a_{i j}\right)$ be an $n \times n$ matrix with prime number entries in the progression $P m+1$. As above, all entries of $A_{p_{i}}$ are $\overline{1}$ and $A_{p_{i}}$ is singular for $1 \leq i \leq k$. Let $A_{11}$ be the $(n-1) \times(n-1)$ minor obtained from $A$ by deleting the first row and the first column of $A$. By the inductive hypothesis, we may assume that $\operatorname{det} A_{11} \neq 0$. Again we may keep all $a_{i j}$ fixed when $i \neq 1, j \neq 1$ and vary $a_{11}$ in the progression $P m+1$. We also have

$$
\operatorname{det} A=a_{11} \operatorname{det} A_{11}+\sum_{j=2}^{n} a_{1 j} \operatorname{det} A_{1 j},
$$

where $A_{1 j}$ is the $(n-1) \times(n-1)$ minor obtained from $A$ by deleting the first row and the $j$ th column of $A$. As $\operatorname{det} A_{11} \neq 0$ and

$$
\sum_{j=2}^{n} a_{1 j} \operatorname{det} A_{1 j}
$$


is fixed, we may vary $a_{11}$ to guarantee that $\operatorname{det} A \neq 0$. Therefore, $A$ is nonsingular and the complexity of $A$ is $\geq p_{k+1}>N$. Lastly, there exist infinitely many such $A$ since there are infinitely many choices for $a_{11}$ as a prime number in the progression $P m+1$. This completes the induction and the proof of Theorem 9 .

\section{Proof of Theorem 1}

The claim trivially holds when $n=1$ so we may assume for the rest of the argument that $n \geq 2$. First note that the number of all $n \times n$ matrices with prime number entries that are $\leq x$ is $\pi(x)^{n^{2}}$. Since $M_{n}(x) \leq \pi(x)^{n^{2}}$, we see that

$$
\limsup _{x \rightarrow \infty} \frac{M_{n}(x)}{\pi(x)^{n^{2}}} \leq 1
$$

Let $p$ be a prime number and let $B=\left(\overline{b_{i j}}\right)$ be a nonsingular matrix over $\mathbb{Z}_{p}$ such that all entries of $B$ are in $\{\overline{1}, \ldots, \overline{p-1}\}$. An important step in the proof is to use the modular interpretation of nonsingularity as in Theorem 8. To this end, consider any matrix $A=\left(a_{i j}\right)$ with prime number entries that are $\leq x$ and satisfying $A_{p}=B$, where the matrix $A_{p}$ is defined as in section 2. As $B$ is assumed to be nonsingular over $\mathbb{Z}_{p}$, Theorem 8 tells us that $A$ is nonsingular. Clearly, each entry of $A$ is a prime number $\leq x$ that lies in a progression with common difference $p$. Precisely,

$$
a_{i j} \equiv b_{i j} \quad(\bmod p)
$$

and $\overline{b_{i j}} \neq \overline{0}$ for all $i, j$. Therefore, by the prime number theorem for arithmetic progressions (see Chap. 20 of [5]), it follows that each entry of $A$ can be chosen in

$$
(1+o(1)) \frac{\pi(x)}{p-1}
$$

many ways as $x \rightarrow \infty$. Consequently, the number of such nonsingular matrices $A$ corresponding to $B$, by reducing entries of $A$ modulo $p$, is

$$
(1+o(1))\left(\frac{\pi(x)}{p-1}\right)^{n^{2}}
$$

as $x \rightarrow \infty$. Next let $N(p)$ be the number of nonsingular matrices over $\mathbb{Z}_{p}$ with entries in $\{\overline{1}, \ldots, \overline{p-1}\}$. As the $A$ 's corresponding to different such $B$ 's have to be different, we infer from (3.2) that

$$
M_{n}(x) \geq(1+o(1))\left(\frac{\pi(x)}{p-1}\right)^{n^{2}} N(p)
$$

as $x \rightarrow \infty$. Now (3.3) further gives that

$$
\liminf _{x \rightarrow \infty} \frac{M_{n}(x)}{\pi(x)^{n^{2}}} \geq \frac{N(p)}{(p-1)^{n^{2}}} .
$$

To complete the proof, we need to find a good lower bound for $N(p)$. Note that each such matrix $B$ as above may be viewed as an invertible linear map belonging to the general linear group $G L_{n}\left(\mathbb{Z}_{p}\right)$. Let $\left\{v_{1}, \ldots, v_{n}\right\}$ be a basis for $\mathbb{Z}_{p}^{n}$ over $\mathbb{Z}_{p}$. It suffices to find a good lower bound for the number invertible linear maps from $\mathbb{Z}_{p}^{n}$ to $\mathbb{Z}_{p}^{n}$, where the allowed coefficients are 
in $\{\overline{1}, \ldots, \overline{p-1}\}$. Let $f: \mathbb{Z}_{p}^{n} \rightarrow \mathbb{Z}_{p}^{n}$ be such a linear map. Then $f$ is determined uniquely by $f\left(v_{1}\right), \ldots, f\left(v_{n}\right)$. Starting with $f\left(v_{1}\right)$, say

$$
f\left(v_{1}\right)=\sum_{i=1}^{n} \overline{x_{i}} v_{i}
$$

with $\overline{x_{i}} \in\{\overline{1}, \ldots, \overline{p-1}\}$. Clearly, $f\left(v_{1}\right) \neq 0$ and $f\left(v_{1}\right)$ can be chosen in $(p-1)^{n}$ different ways. In general, for $2 \leq j \leq n, f\left(v_{j}\right)$ can not be a $\mathbb{Z}_{p}$ combination of $f\left(v_{1}\right), \ldots, f\left(v_{j-1}\right)$. Observe that

$$
\left|\left\{\overline{\alpha_{1}} f\left(v_{1}\right)+\cdots+\overline{\alpha_{j-1}} f\left(v_{j-1}\right): \overline{\alpha_{1}}, \ldots, \overline{\alpha_{j-1}} \in \mathbb{Z}_{p}\right\}\right|=p^{j-1}
$$

as $\left\{v_{1}, \ldots, v_{j-1}\right\}$ are already chosen to be linearly independent. Therefore, the number of choices for $f\left(v_{j}\right)$, so as to make $f$ invertible, is at least

$$
(p-1)^{n}-p^{j-1} \text {. }
$$

Let us remark here that this is far from being an exact count for the number of choices of $f\left(v_{j}\right)$ since some of the combinations of $f\left(v_{1}\right), \ldots, f\left(v_{j-1}\right)$ in the form

$$
\overline{\alpha_{1}} f\left(v_{1}\right)+\cdots+\overline{\alpha_{j-1}} f\left(v_{j-1}\right)
$$

with $\overline{\alpha_{1}}, \ldots, \overline{\alpha_{j-1}} \in \mathbb{Z}_{p}$ can be written as a combination of $v_{1}, \ldots, v_{n}$, where some of the coefficients are $\overline{0}$. It follows that the number of such invertible linear maps is at least

$$
(p-1)^{n} \prod_{j=2}^{n}\left((p-1)^{n}-p^{j-1}\right)
$$

which amounts to the lower bound

$$
N(p) \geq(p-1)^{n} \prod_{j=2}^{n}\left((p-1)^{n}-p^{j-1}\right) .
$$

It is worth pointing out that (3.5) is nontrivial only when $p$ is large enough in terms of $n$. Combining (3.4) with (3.5), we deduce that

$$
\liminf _{x \rightarrow \infty} \frac{M_{n}(x)}{\pi(x)^{n^{2}}} \geq(p-1)^{n-n^{2}} \prod_{j=2}^{n}\left((p-1)^{n}-p^{j-1}\right) .
$$

We also have

$$
(p-1)^{n-n^{2}} \prod_{j=2}^{n}\left((p-1)^{n}-p^{j-1}\right)=\prod_{j=2}^{n}\left[1-\frac{1}{p^{n-j+1}\left(1-\frac{1}{p}\right)^{n}}\right],
$$

where the product over $j$ on the right hand side of (3.7) is nonempty since $n \geq 2$ is assumed. Moreover, $n-j+1 \geq 1$ holds for all $2 \leq j \leq n$ and using the fact that $p$ can be arbitrarily large, one easily sees that

$$
\lim _{p \rightarrow \infty} \prod_{j=2}^{n}\left[1-\frac{1}{p^{n-j+1}\left(1-\frac{1}{p}\right)^{n}}\right]=1 .
$$

As a result of (3.6)-(3.8), we have

$$
\liminf _{x \rightarrow \infty} \frac{M_{n}(x)}{\pi(x)^{n^{2}}}=1
$$


Finally assembling (3.1) with (3.9), one completes the proof of Theorem 1.

\section{Proof of TheOREM 2}

First assume that $A$ is similar to such a matrix $B$ with respect to an orthonormal basis of $\mathbb{R}^{n}$. Then there exists a real unitary matrix $P$ satisfying $P^{-1} A P=B$ and $P^{-1}=P^{t}$. It is easy to see that

$$
\operatorname{det} B= \pm \prod_{i=1}^{k}\left(\cos ^{2} \theta_{i}+\sin ^{2} \theta_{i}\right)= \pm 1
$$

for some $k \leq[n / 2]$, where $k$ is the number of $2 \times 2$ blocks appearing on the main diagonal of $B$. Note that $\operatorname{det} A=\operatorname{det} B$ and $|\operatorname{det} A|=1$ follows from (4.1). Moreover, we have $\operatorname{tr}\left(B B^{t}\right)=n$. Using the facts that $P$ is real unitary and

$$
P^{t} A^{t}\left(P^{-1}\right)^{t}=B^{t}
$$

we infer that

$$
P^{-1} A A^{t} P=P^{-1} A A^{t}\left(P^{-1}\right)^{t}=P^{-1} A P P^{t} A^{t}\left(P^{-1}\right)^{t}=B B^{t} .
$$

Thus by (4.2), $A A^{t}$ is similar to $B B^{t}$ and we obtain that $\operatorname{tr}\left(A A^{t}\right)=\operatorname{tr}\left(B B^{t}\right)=n$. This completes the proof of the necessity part of the claim. For the sufficiency part of the claim, assume that $|\operatorname{det} A|=1$ and $\operatorname{tr}\left(A A^{t}\right)=n$. Note that $A A^{t}$ is a symmetric matrix. Let $\lambda$ be an eigenvalue of $A A^{t}$. It is well-known that eigenvalues of symmetric matrices are real so that $\lambda$ is real. Moreover, if $v \neq 0$ is an eigenvector for $\lambda$, then using $A A^{t} v=\lambda v$, one gets

$$
\left\langle A A^{t} v, v\right\rangle=\langle\lambda v, v\rangle=\lambda\langle v, v\rangle \text {. }
$$

Also

$$
\left\langle A A^{t} v, v\right\rangle=\left\langle A^{t} v, A^{t} v\right\rangle \geq 0
$$

holds as $\langle.,$.$\rangle is positive definite. Clearly, A A^{t}$ is nonsingular and $\langle v, v\rangle>0$. Then comparison of (4.3) and (4.4) tells us that all eigenvalues of $A A^{t}$ are positive real numbers. Let $\lambda_{1}, \ldots, \lambda_{n}$ be these eigenvalues. But we know that

$$
n=\operatorname{tr}\left(A A^{t}\right)=\sum_{i=1}^{n} \lambda_{i}
$$

and as $A$ is real matrix with $|\operatorname{det} A|=1$, we also have that

$$
1=(\operatorname{det} A)^{2}=\operatorname{det} A A^{t}=\prod_{i=1}^{n} \lambda_{i} .
$$

Gathering (4.5) and (4.6), one sees that

$$
\frac{1}{n} \sum_{i=1}^{n} \lambda_{i}=\left(\prod_{i=1}^{n} \lambda_{i}\right)^{\frac{1}{n}}
$$

where $\lambda_{1}, \ldots, \lambda_{n}$ are positive real numbers. (4.7) represents the equality case of the arithmeticgeometric mean inequality and it is well-known that this can happen only when

$$
\lambda_{1}=\cdots=\lambda_{n}=\lambda
$$


for some $\lambda>0$. From (4.6) and (4.8), one infers that $\lambda^{n}=1$ and this forces $\lambda=1$. As there exists a basis $\left\{v_{1}, \ldots, v_{n}\right\}$ of $\mathbb{R}^{n}$ consisting of eigenvectors of $A A^{t}$ (see Theorem 4.3 on p. 219 of [13]) and all eigenvalues of $A A^{t}$ are $1, A A^{t}$ fixes all of the basis elements. This shows that $A A^{t}=I$. Therefore, $A$ is real unitary and $A$ has to be similar to the desired block matrix $B$ with respect to an orthonormal basis arising from $\langle.,$.$\rangle (see Theorem 6.4$ on p. 230 of [13]). This completes the proof of Theorem 2.

\section{Proof of Theorem 3}

For the necessity part of the claim, note that

$$
\operatorname{det} B=e^{i \sum \theta_{k}} \text { and } \operatorname{tr}\left(B B^{*}\right)=n,
$$

where $e^{i \theta_{k}}, 1 \leq k \leq n$ are the diagonal entries of $B$. Moreover, there exists a complex unitary matrix $U$ such that

$$
U^{-1} A U=B \text { and } U^{-1}=U^{*} .
$$

First from (5.1) and (5.2), $|\operatorname{det} A|=|\operatorname{det} B|=1$ follows. Similarly as in the proof of Theorem 2 , we can show, using (5.1) and (5.2), that $\operatorname{tr}\left(A A^{*}\right)=\operatorname{tr}\left(B B^{*}\right)=n$. The sufficiency part of the claim can be shown similarly as in Theorem 2 by noting that $A A^{*}$ is hermitian and has positive real eigenvalues. Thus the equality case of the arithmetic-geometric mean inequality is again applicable. In this way, one may deduce that $A$ is complex unitary. Finally, using Theorem 6.2 on p. 228 of [13], we see that $A$ has to be similar to such a matrix $B$ with respect to an orthonormal basis arising from the hermitian product $\langle.,$.$\rangle . This completes the proof$ of Theorem 3.

\section{Proof of Corollary 1}

Assume that $A$ is complex unitary with eigenvalues $\lambda_{1}, \ldots, \lambda_{n}$. Since $\operatorname{det} A^{*}=\overline{\operatorname{det} A}$ (here of course $\overline{\operatorname{det} A}$ is the complex conjugate of $\operatorname{det} A$, unlike its meaning in section 2 ), we have $|\operatorname{det} A|=1$. Moreover, all eigenvalues of $A$ are of the form $e^{i \theta}$ for some real number $\theta$ so that

$$
\sum_{i=1}^{n}\left|\lambda_{i}\right|=n
$$

holds. This completes the necessity part of the claim. For the sufficiency part of the claim, assume that

$$
|\operatorname{det} A|=1 \text { and } \sum_{i=1}^{n}\left|\lambda_{i}\right|=n \text {. }
$$

Since $A$ is a normal matrix, by the spectral theorem (see p. 227 of [13]), there exists a complex unitary matrix $U$ such that

$$
U^{-1} A U=B,
$$

where $B$ is a diagonal matrix consisting of eigenvalues of $A$ on the main diagonal. From (6.1), we see that

$$
\prod_{i=1}^{n}\left|\lambda_{i}\right|=|\operatorname{det} A|=1
$$


and consequently that

$$
\frac{1}{n} \sum_{i=1}^{n}\left|\lambda_{i}\right|=1=\left(\prod_{i=1}^{n}\left|\lambda_{i}\right|\right)^{\frac{1}{n}}
$$

By the equality case of the arithmetic-geometric mean inequality, (6.3) implies that $\left|\lambda_{i}\right|=1$ for all $i$. Therefore, the diagonal entries of $B$ are of the form $e^{i \theta}$ and $B$ is complex unitary. As complex unitary matrices form a group under multiplication and $U$ is complex unitary, one obtains from (6.2) that $A=U B U^{-1}$ is complex unitary as well. This completes the proof of Corollary 1.

\section{Proof of Theorem 4}

First assume that $c N$ is unitary for some positive number $c$. Then

$$
c N c N^{*}=c^{2} N N^{*}=I
$$

and it follows from (7.1) that

$$
\operatorname{det} N N^{*}=\frac{1}{c^{2 n}} \text { and } \operatorname{tr}\left(N N^{*}\right)=\frac{n}{c^{2}} \text {. }
$$

But we also have $\operatorname{det} N N^{*}=\operatorname{det} N \overline{\operatorname{det} N}=|\operatorname{det} N|^{2}$ so that

$$
|\operatorname{det} N|^{\frac{2}{n}}=\frac{1}{c^{2}}
$$

follows from (7.2). Gathering (7.2) and (7.3), (1.1) is obtained. Conversely, assume that (1.1) holds. Since $N$ is nonsingular, $N N^{*}$ is a positive definite hermitian matrix. Therefore, by the spectral theorem (see Theorem 5.3 on p. 226 of [13]), $N N^{*}$ is similar to a diagonal matrix consisting of the positive eigenvalues of $N N^{*}$ on the main diagonal. Using this, we see that

$$
|\operatorname{det} N|^{2}=\operatorname{det} N N^{*}=\prod_{i=1}^{n} \lambda_{i} \text { and } \operatorname{tr}\left(N N^{*}\right)=\sum_{i=1}^{n} \lambda_{i},
$$

where $\lambda_{1}, \ldots, \lambda_{n}$ are the positive eigenvalues of $N N^{*}$. As a result of (7.4), (1.1) can be written in the form

$$
\frac{1}{n} \sum_{i=1}^{n} \lambda_{i}=\left(\prod_{i=1}^{n} \lambda_{i}\right)^{\frac{1}{n}}
$$

Once again, (7.5) gives the equality case of the arithmetic-geometric mean inequality and one concludes that $\lambda_{i}=\lambda>0$ for all $i$. Finally, $N N^{*}$ is similar to the diagonal matrix $\lambda I$ and we get that

$$
\left(\frac{1}{\sqrt{\lambda}} N\right)\left(\frac{1}{\sqrt{\lambda}} N^{*}\right)=I
$$

Consequently from (7.6), $c N$ is unitary with $c=\frac{1}{\sqrt{\lambda}}>0$ and this completes the proof of Theorem 4. 


\section{Proof of TheOREM 5}

As $A$ and $B$ are commuting complex matrices, we may use a special case of a classical theorem of Frobenius on the simultaneous triangulation of an arbitrary set of commuting matrices by unitary similarity. For a nice treatment and proof of this result based on representation theory, the reader is referred to the paper of Newman [15]. Precisely, by Theorem 1 of [15], there exists a unitary matrix $S$ such that

$$
U_{1}=S^{*} A S \text { and } U_{2}=S^{*} B S
$$

are both upper triangular matrices. Therefore, $A$ and $B$ can be simultaneously put into triangular form with respect to an orthonormal basis of $\mathbb{C}^{n}$. Using (8.1), one obtains that

$$
\operatorname{tr}\left(U_{1} U_{2}\right)=\operatorname{tr}\left(S^{*} A S S^{*} B S\right)=\operatorname{tr}\left(S^{*} A B S\right)=\operatorname{tr}(A B) .
$$

Again from (8.1), we also have

$$
\operatorname{tr}\left(U_{1}^{2}\right)=\operatorname{tr}\left(S^{*} A^{2} S\right)=\operatorname{tr}\left(A^{2}\right) \text { and } \operatorname{tr}\left(U_{2}^{2}\right)=\operatorname{tr}\left(S^{*} B^{2} S\right)=\operatorname{tr}\left(B^{2}\right) .
$$

Assume now that (1.2) holds for $A$ and $B$. Then combining (8.2) with (8.3), we see that

$$
\left(\operatorname{tr}\left(U_{1} U_{2}\right)\right)^{2}=\operatorname{tr}\left(U_{1}^{2}\right) \operatorname{tr}\left(U_{2}^{2}\right) .
$$

Observe that $U_{1}$ and $U_{2}$ are upper triangular matrices whose main diagonals consist of eigenvalues of $A$ and $B$, respectively. Let $\lambda_{1}, \ldots, \lambda_{n}$ be the eigenvalues of $A$ and let $\mu_{1}, \ldots, \mu_{n}$ be the eigenvalues of $B$. By assumption, $\lambda_{i}$ and $\mu_{i}$ are all nonzero real numbers. Moreover, $U_{1} U_{2}$ is also an upper triangular matrix whose main diagonal consists of the numbers $\lambda_{i} \mu_{i}$. It follows that

$$
\left(\operatorname{tr}\left(U_{1} U_{2}\right)\right)^{2}=\left(\sum_{i=1}^{n} \lambda_{i} \mu_{i}\right)^{2} .
$$

By similar reasoning, one further infers that

$$
\operatorname{tr}\left(U_{1}^{2}\right)=\sum_{i=1}^{n} \lambda_{i}^{2} \text { and } \operatorname{tr}\left(U_{2}^{2}\right)=\sum_{i=1}^{n} \mu_{i}^{2}
$$

Assembling (8.4)-(8.6), we deduce that

$$
\left(\sum_{i=1}^{n} \lambda_{i} \mu_{i}\right)^{2}=\left(\sum_{i=1}^{n} \lambda_{i}^{2}\right)\left(\sum_{i=1}^{n} \mu_{i}^{2}\right) .
$$

Clearly, (8.7) represents the equality case of the Cauchy-Schwarz inequality. Then it is wellknown that

$$
\mu_{i}=c \lambda_{i}
$$

holds for all $i$, where

$$
c=\left(\frac{\sum_{i=1}^{n} \mu_{i}^{2}}{\sum_{i=1}^{n} \lambda_{i}^{2}}\right)^{\frac{1}{2}}>0 .
$$

From (1.2), we have

$$
\prod_{i=1}^{n} \lambda_{i}=\operatorname{det} A=\operatorname{det} B=\prod_{i=1}^{n} \mu_{i} .
$$


Consequently from (8.8)-(8.10) and the fact that $A$ and $B$ are nonsingular, one obtains $c^{n}=1$ and $c=1$. Therefore, $A$ and $B$ have the same eigenvalues, counting multiplicity, so have the same characteristic polynomial. Conversely, if $A$ and $B$ have the same characteristic polynomial so have the same eigenvalues, counting multiplicity, say $\lambda_{1}, \ldots, \lambda_{n}$, then we have

$$
\operatorname{det} A=\prod_{i=1}^{n} \lambda_{i}=\operatorname{det} B \text {. }
$$

Moreover, by (8.2) and (8.3),

$$
(\operatorname{tr}(A B))^{2}=\left(\operatorname{tr}\left(U_{1} U_{2}\right)\right)^{2}=\left(\sum_{i=1}^{n} \lambda_{i}^{2}\right)^{2}=\operatorname{tr}\left(U_{1}^{2}\right) \operatorname{tr}\left(U_{2}^{2}\right)=\operatorname{tr}\left(A^{2}\right) \operatorname{tr}\left(B^{2}\right)
$$

holds. From (8.11) and (8.12), one completes the proof of Theorem 5.

\section{Proof of Theorem 6}

The necessity part of the claim is obvious. For the sufficiency part, assume that $A$ and $A^{*}$ have the same eigenvectors. Let us show by induction on the size of the matrices that $A$ and $A^{*}$ are simultaneously diagonalizable. The base case of the induction clearly holds for $1 \times 1$ matrices $A$ and $A^{*}$. Thus for the inductive step, we may assume that $A$ and $A^{*}$ are $n \times n$ matrices with $n>1$. Since $A$ is a complex matrix, it has an eigenvalue, say $\lambda$. Then $A v_{1}=\lambda v_{1}$ holds for some unit vector $v_{1}$. Let

$$
W=\left\{c v_{1}: c \in \mathbb{C}\right\}
$$

be the complex space spanned by $v_{1}$. Also let $\langle.,$.$\rangle be the standard positive definite her-$ mitian product on $\mathbb{C}^{n}$ so that for any two $v=\left(a_{1}, \ldots, a_{n}\right), w=\left(b_{1}, \ldots, b_{n}\right)$ in $\mathbb{C}^{n}$, we have

$$
\langle v, w\rangle=\sum_{i=1}^{n} a_{i} \overline{b_{i}}
$$

where $\overline{b_{i}}$ is the complex conjugate of $b_{i}$. In this setting, one gets by the Gram-Schmidt orthogonalization process that

$$
W \oplus W^{\perp}=\mathbb{C}^{n},
$$

where $W^{\perp}$ is the orthogonal complement of $W$ in $\mathbb{C}^{n}$ with respect to the standard hermitian product. Note that

$$
\operatorname{dim}_{\mathbb{C}} W^{\perp}=n-1
$$

follows from (9.1). Let us see that $W^{\perp}$ is stable under $A$. To this end, take any $w \in W^{\perp}$. Then $\left\langle c v_{1}, w\right\rangle=0$ for all $c \in \mathbb{C}$. As $A$ and $A^{*}$ have the same eigenvectors, $v_{1}$ is an eigenvector of $A^{*}$ and $A^{*} v_{1}=\lambda_{1} v_{1}$ holds for some eigenvalue $\lambda_{1}$ of $A^{*}$. Then we observe that

$$
\left\langle c v_{1}, A w\right\rangle=\left\langle c A^{*} v_{1}, w\right\rangle=\left\langle c \lambda_{1} v_{1}, w\right\rangle=0 .
$$

Therefore, from (9.3), $A w \in W^{\perp}$ follows and $W^{\perp}$ is stable under $A$. Similarly, one can show that $W^{\perp}$ is also stable under $A^{*}$. By (9.2), the inductive hypothesis holds for $W^{\perp}$ and $A$ and $A^{*}$ are simultaneously diagonalizable over $W^{\perp}$. This means that there exists an orthonormal basis $\mathcal{B}$ of $W^{\perp}$ such that $A$ and $A^{*}$ are both diagonal with respect to $\mathcal{B}$. But then $A$ and 
$A^{*}$ are both diagonal over $\mathbb{C}^{n}$ with respect to $\mathcal{B} \cup\left\{v_{1}\right\}$. Next let $v$ be any basis element in $\mathcal{B} \cup\left\{v_{1}\right\}$. Then note that since all eigenvalues of $A$ are real,

$$
A v=\mu_{1} v \text { and } A^{*} v=\mu_{2} v
$$

holds with real $\mu_{1}$. Using (9.4) and the properties of the hermitian product, one may deduce that

$$
\mu_{2}\langle v, v\rangle=\left\langle A^{*} v, v\right\rangle=\langle v, A v\rangle=\overline{\mu_{1}}\langle v, v\rangle=\mu_{1}\langle v, v\rangle .
$$

Since $\langle v, v\rangle>0$, we obtain from (9.4) and (9.5) that $\mu_{1}=\mu_{2}$ and consequently that

$$
A v=A^{*} v
$$

for all $v \in \mathcal{B} \cup\left\{v_{1}\right\}$. Thus $A=A^{*}$ and $A$ is hermitian. This completes the proof of Theorem 6 .

\section{Proof of Theorem 7}

The necessity part of the claim is obvious. For the sufficiency part, assume that $A A^{*}$ and $A^{*} A$ have the same eigenvectors corresponding to every common eigenvalue of $A A^{*}$ and $A^{*} A$. Note that $A A^{*}$ is a hermitian matrix so by the spectral theorem (see Theorem 5.3 on p. 226 of [13]), it is diagonalizable. It follows that there exists a basis $\mathcal{B}$ of $\mathbb{C}^{n}$ consisting of the eigenvectors of $A A^{*}$. Let $v$ be any basis element of $\mathcal{B}$. Then $v$ is an eigenvector of $A A^{*}$ so that

$$
A A^{*} v=\lambda v
$$

for some real number $\lambda$. Note that $A A^{*}$ and $A^{*} A$ have the same eigenvalues so that $\lambda$ is also an eigenvalue of $A^{*} A$. Thus by assumption, $v$ is also an eigenvector of $A^{*} A$ corresponding to the common eigenvalue $\lambda$. It follows that

$$
A^{*} A v=\lambda v=A A^{*} v .
$$

As $v \in \mathcal{B}$ is arbitrary, we conclude that $A A^{*}=A^{*} A$ and $A$ is therefore normal. This completes the proof of Theorem 7 .

\section{REFERENCES}

[1] E. Alkan, Multiplicative number theory with applications to modular forms and enumeration of groups, Ph. D. Thesis, University of Wisconsin, Madison, 2003.

[2] E. Alkan, Nonvanishing of Fourier coefficients of modular forms, Proc. Amer. Math. Soc. 131 (2003), 1673-1680.

[3] E. Alkan, On the sizes of gaps in the Fourier expansion of modular forms, Canad. J. Math. 57 (2005), 449-470.

[4] A. Dănescu, V. Vâjâitu, A. Zaharescu, Unimodular matrices whose entries are squares of those of a unimodular matrix, Rev. Roumaine Math. Pures Appl. 46 (2001), 419-430.

[5] H. Davenport, Multiplicative Number Theory, third ed., Grad. Texts in Math., vol. 74, Springer-Verlag, New York, 2000.

[6] P. Erdös, A. Wintner, Additive arithmetical functions and statistical independence, Amer. J. Math 61 (1939), 713-721.

[7] P. Erdös, M. Kac, The Gaussian law of errors in the theory of additive number theoretic functions, Amer. J. Math 62 (1940), 738-742.

[8] D. J. Griffiths, Introduction to Quantum Mechanics, second ed., Pearson, Prentice Hall, 2005.

[9] G. H. Hardy, S. Ramanujan, The normal order of prime factors of a number n, Quart. J. Math. 48 (1917), 76-92.

[10] A. J. Hoffman, O. Taussky, A characterizarion of normal matrices, J. Res. Nat. Bur. Standards 52 (1954), $17-19$. 
[11] J. Komlós, On the determinant of $(0,1)$ matrices, Studia Sci. Math. Hungar 2 (1967), 7-21.

[12] J. Komlós, On the determinant of random matrices, Studia Sci. Math. Hungar 3 (1968), 387-399.

[13] S. Lang, Linear Algebra, Undergrad. Texts in Math., third ed., Springer, New York, 1987.

[14] J. Neukirch, Algebraic Number Theory, Grundlehren Math. Wiss. (A Series of Comprehensive Studies in Mathematics), vol. 322, Springer-Verlag, Berlin, 1999.

[15] M. Newman, Two classical theorems on commuting matrices, J. Res. Nat. Bur. Standards Sect. B 71B (1967), 69-71.

[16] J. P. Serre, A Course in Arithmetic, Grad. Texts in Math., vol. 7, Springer-Verlag, New York, 1973.

Department of Mathematics, Koç University, Rumelifeneri Yolu, 34450, Sarıer, Istanbul, TURKEY. e-mail: ealkan@ku.edu.tr

Department of Physics, Koç University, Rumelifeneri Yolu, 34450, Sarıyer, Istanbul, TURKEY. e-mail: eyoruk13@ku.edu.tr 